\title{
PAPER
}

\section{Ocular motor myotonic phenomenon in myotonic dystrophy}

\author{
M Versino, B Rossi, G Beltrami, G Sandrini, V Cosi
}

J Neurol Neurosurg Psychiatry 2002;72:236-240

See end of article for authors' affiliations

....................

Correspondence to:

Dr M Versino, Dipartimento

Scienze Neurologiche

Università di Pavia,

Fondazione Istituto

Neurologico C Mondino,

IRCCS, Via Palestro 3,

27100 Pavia, Italy;

mversino@unipv.it

Received 18 April 2001

In revised form

28 August 2001

Accepted

23 October 2001 alities and a possible extraocular muscle myo-

Objective: To detect disconjugate ocular motor abnormalities
tonic phenomenon in patients with myotonic dystrophy (MyD).

Methods: The magnetic scleral search coil technique was used to record monocularly the small $\left(25^{\circ}\right)$ and large $\left(50^{\circ}\right)$ saccades, which were paced to two interstimulus intervals (ISIs), one short (1 s), the other long $(5 \mathrm{~s})$. The case study comprised 20 patients with MyD, 10 patients with multiple sclerosis (MS), and 10 controls. The amplitude, duration, peak velocity, and skewness of the velocity profile (ratio between the acceleration and the deceleration periods) of each saccade were measured. The disconjugate parameters (difference between the two eyes of the same measure), and the myotonic parameter (the maximal (as absolute value) short-long ISI difference between the same measures) were considered.

Results: The disconjugate parameters were the same in all three groups. The mean values of myotonic parameters found in patients with MyD for duration (for both small and large target displacements) and skewness (for small target displacements only) differed from those found for both the MS and the control groups. Additionally, the occurrence of individual patients presenting with abnormal duration and skewness parameters was higher in the MyD than in the MS group. In patients with MyD, the saccade duration was longer for long than for short ISI; the effect derived from a prolongation of the acceleration period, which manifested as an increase in skewness.

Conclusion: The results can be explained by a combination of the myotonic and the warm up phenomena. A delay in the relaxation (myotonia) of the extraocular muscle may be more evident after a long fixation period (long ISI) and it may improve by increasing saccade pacing (short ISI-warm up). This phenomenon is slight, and is unlikely to affect saccade performance significantly, but it may provide some insight into the nature of the disorder affecting extraocular and skeletal muscles in myotonic dystrophy.
M yotonic dystrophy is an autosomal dominant multisystem disorder that derives from an unstable CTG repeat located on chromosome 19. ${ }^{12}$

Skeletal muscles may present dystrophic changes, which mainly involve temporal, facial, and distal limb muscles, and a delay in muscle relaxation after contraction. This delay is the defining feature of the myotonic phenomenon, or myotonia. ${ }^{3}$ Several and repetitive muscle contractions may momentarily reduce myotonia in the given muscle, and this has been called the warm up phenomenon. ${ }^{5}$

Patients with MyD rarely complain of, or present with, ocular motor symptoms or signs other than lid ptosis. By contrast, eye movement recordings have disclosed several abnormalities, including inaccurate and slowed saccades, ${ }^{6-11}$ but no data are available about the yoking of the eyes during saccades, or about the possibility that saccade abnormalities may be disconjugate. Whether these abnormalities are attributable to the ocular motor structures within the CNS, or to the extraocular muscles, or both, is still a matter of debate, and it is possible that the pathophysiology of ocular motor abnormalities differs from patient to patient. ${ }^{10}$

When ocular motor abnormalities are considered to be the consequence of an extraocular muscle dysfunction, they are usually attributed to an extraocular myopathy. Although myotonic discharges are electromyographically detectable in extraocular muscles, ${ }^{12}$ to our knowlegde only one case report has considered the question of the possible occurrence of extraocular myotonia. ${ }^{13}$

The aims of the present report are to detect whether saccade abnormalities involve saccade yoking-namely, disconjugate parameters-and to demonstrate that saccadic abnormalities may be related both to the extraocular myotonia and to the warm up phenomenon.

\section{MATERIALS AND METHODS \\ Patients}

We enlisted 20 patients with MyD (mean age 41.2 years; age range 15-68 years) derived from 17 families, 10 patients with multiple sclerosis (MS; mean age 35.6 years; age range $20-57$ years), and 10 controls (mean age 39.5 years; age range 22-65 years). All participants gave their informed consent to eye movement recording.

All patients showed visual acuity above $7 / 10$ in both eyes, and none of them complained of diplopia or presented with clinically evident ocular motor signs, other than lid ptosis.

We included the MS group because their ocular motor abnormalities are likely to be attributable to a CNS rather than to an extraocular muscle dysfunction.

\section{Eye movement recording}

Using a scleral search coil technique (Skalar 3010 system), we recorded monocularly saccadic eye movements simultaneously from both eyes in binocular vision after the signal had been calibrated monocularly in monocular vision. The ocular motor signal was sampled at $250 \mathrm{~Hz}$ ( 12 bit analog to digital converter resolution), filtered with a band pass from 0 to $70 \mathrm{~Hz}$ and stored on magnetic support for subsequent analyses.

Abbreviations: $M y D$, myotonic dystrophy; MS, multiple sclerosis; ISIs, interstimulus intervals 


\section{Saccade paradigms}

We used four different paradigms in all subjects. In the first two paradigms, the targets were light emitting diodes in the $0^{\circ},+25^{\circ}$ (right), and $-25^{\circ}$ (left) positions, $104 \mathrm{~cm}$ from the subject. The target in primary position was activated for $1 \mathrm{sec}-$ ond and then switched off, whereupon a target in a lateral position was lit either for one (paradigm 1) or for 5 seconds (paradigm 2), before the target in primary position was relit. The targets in lateral positions were activated 15 times in a pseudorandom sequence.

In the other two paradigms we only used the light emitting diodes in the $+25^{\circ}$ and $-25^{\circ}$ positions, and lit them alternately 15 times each. The activation period was either 1 second (paradigm 3) or 5 seconds (paradigm 4).

Overall, we used two different target displacements (small $\left(25^{\circ}\right)$ and large $\left.\left(50^{\circ}\right)\right)$, and two different interstimulus intervals (ISIs) (short (1s) and long ( $5 \mathrm{~s}$ ). Accordingly, we labelled paradigm 1 as small amplitude and short ISI, paradigm 2 as small amplitude and long ISI, paradigm 3 as large amplitude and short ISI, and paradigm 4 as large amplitude and long ISI.

The first two (small amplitude) paradigms consisted of four saccade directions - that is, rightward centrifugal and centripetal and leftward centrifugal and centripetal, whereas the last two paradigms (large amplitude) consisted of two saccade directions - that is, rightward and leftward.

\section{Eye movement parameters}

Saccades were identified by means of an interactive ad hoc software.

We measured the amplitude, the duration, and the peak velocity of each saccade the beginning and the end of which were identified on the basis of velocity threshold criteria $(25 \%$ s). Moreover, we computed the skewness of the velocity profile-namely, the ratio of the duration of the acceleration period (the time elapsing between the beginning of the saccade and the peak velocity instant) to the duration of the deceleration period (the time elapsing between the peak velocity instant and the end of the saccade); acceleration periods longer than deceleration periods lead to skewness greater than 1 , whereas acceleration periods shorter than deceleration periods lead to skewness smaller than 1 .

For each patient, for each eye, for each paradigm, and for each saccade direction, we computed the mean value of each parameter.

\section{Statistical analyses Monocular parameters}

For each subject and for each eye, we compared the amplitude, duration, peak velocity, and skewness mean values for the myotonic and control groups; we used analysis of variance (ANOVA) (group variable: diagnosis, two levels: MyD and control). Although target displacement was constant throughout each paradigm, the duration, the peak velocity, and the skewness are related to and, for this reason, were divided by the corresponding actual eye displacement (amplitude) values.

\section{Disconjugate parameters}

For each subject, for each eye, for each paradigm, and for each saccade direction, we considered the disconjugate amplitude, duration, peak velocity, and skewness computed as the difference between the corresponding values of the eyes divided by the mean of these two values.

The disconjugate parameter mean values were compared by ANOVA (group variable: diagnosis, three levels: MyD, MS, and control).

\section{Myotonic parameters}

The evaluation of myotonic parameters was based on the hypothesis that short but not long ISIs would promote the warm up phenomenon.
Accordingly, for each subject and for each eye parameter, we computed the difference between short and long ISIs for corresponding parameters divided by the average of the two values. We considered both saccade directions for the large amplitude paradigms, and centripetal saccade directions alone for the small amplitude paradigms. Finally, as the myotonic phenomenon is likely to involve the extraocular muscles on an individual basis, for each subject we considered the largest (as an absolute value) ISI difference with its sign among the four available (two eyes and two saccade directions) for each small and large amplitude paradigm. The myotonic, MS, and control group mean values for maximal ISI difference were compared by ANOVA (group variable: diagnosis, three levels: MyD, MS, and control) and, when ANOVA showed a statistically significant difference between the means of the three groups, we also performed contrast analysis. This procedure enabled separate comparison of mean values of myotonic parameters with those collected for the other two groups. Accordingly, we performed two contrasts: contrast 1 compared the MyD with the MS group whereas contrast 2 compared the MyD with the control group.

We also evaluated the patients with MyD and those with MS on an individual basis and to do this, we defined a normal range for maximal ISI difference as the control mean values \pm 2 SD. Then we compared the occurrence of abnormal values in the two groups by Fisher's exact test.

The SPSS/PC+ program was used for all the analyses and the significance level was set at $\mathrm{p}=0.05$.

\section{RESULTS}

\section{Monocular parameters}

Saccade duration and peak velocity, but not saccade amplitude and skewness mean values, were significantly different for all target amplitudes, ISIs, and saccade directions in both eyes. Duration was invariably longer, and peak velocity lower in the MyD than in the control group.

\section{Disconjugate parameters}

None of the mean values in disconjugate parameters in the MyD group differed from those both in the MS group (despite two patients presenting with subclinical internuclear ophthalmoplegia) and in the control group. This held true for all target amplitudes and directions, and for both short and long ISIs. The next section will report the results of additional analyses on disconjugate ocular motor phenomena possibly induced by the occurrence of the myotonic phenomenon.

\section{Myotonic parameters}

Table 1 reports the mean values for the myotonic parameters.

The maximal difference in short-long ISI mean values for saccade duration were invariably negative-that is, durations were greater for long than for short ISIs. The mean values for the three groups were significantly different both for small $(F 2,37=3.33, \mathrm{p}=0.047)$ and for large $(F 2,37=7.32, \mathrm{p}=0.002)$ amplitudes, and contrast analysis showed that the MyD mean value was larger than those of the MS group (contrast 1 : $t 37=2.13, p=0.04$ for small saccades; $t 37=2.21, p=0.034$ for large saccades) and of the control group (contrast 2 : $t 37=2.1$, $\mathrm{p}=0.045$ for small saccades; $t 37=3.68, \mathrm{p}=0.001$ for large saccades) for both target displacements.

The maximal mean values of the short-long ISI difference for saccade skewness were negative. In other words acceleration duration was greater than the deceleration duration for long ISIs than it was for short ISIs: in the MyD group, this was the case both for small and for large target displacements, whereas in the MS group, it was the case for large but not for small saccades; moreover the skewness maximal differences were positive in the control group. The mean values for the three groups differed significantly for small $(F 2,37=6.94$, $\mathrm{p}=0.003)$ but not for large $(F 2,37=2.59, \mathrm{p}=0.089)$ amplitudes. For small target displacements, contrast analysis 
Table 1 Mean (SEM) values for the myotonic parameters and for small and large target displacements, as computed for the myotonic (MyD), multiple sclerosis (MS), and control groups

\begin{tabular}{|c|c|c|c|c|}
\hline Myotonic parameter & $\begin{array}{l}\text { Target } \\
\text { displacement }\end{array}$ & Patient group & Mean & (SEM) \\
\hline \multirow[t]{6}{*}{ Duration $\times 10^{3}$} & \multirow[t]{3}{*}{ Small } & MyD & -942.210 & 172.55 \\
\hline & & MS & -423.55 & 183.16 \\
\hline & & Control & -436.98 & 51.89 \\
\hline & \multirow[t]{3}{*}{ Large } & MyD & -1072.380 & 139.72 \\
\hline & & MS & -634.02 & 127.18 \\
\hline & & Control & -340.35 & 98.53 \\
\hline \multirow[t]{6}{*}{ Peak velocity $\times 10^{3}$} & \multirow[t]{3}{*}{ Small } & MyD & 2311.07 & 169.81 \\
\hline & & MS & 1674.71 & 321.54 \\
\hline & & Control & 1946.07 & 268.32 \\
\hline & \multirow[t]{3}{*}{ Large } & MyD & 1694.17 & 140.72 \\
\hline & & MS & 2060.96 & 352.21 \\
\hline & & Control & 1270.99 & 234.12 \\
\hline \multirow[t]{6}{*}{ Skewness $\times 10^{3}$} & \multirow[t]{3}{*}{ Small } & MyD & -9.25 & 2.82 \\
\hline & & MS & 3.21 & 2.23 \\
\hline & & Control & 1.99 & 1.75 \\
\hline & \multirow[t]{3}{*}{ Large } & MyD & -3.88 & 1.20 \\
\hline & & MS & -1.32 & 1.09 \\
\hline & & Comtrol & 0.09 & 1.00 \\
\hline \multicolumn{5}{|c|}{$\begin{array}{l}\text { The myotonic parameters were chosen for each given subject among those computed for each eye and for } \\
\text { each saccade direction and separately for small and large amplitude paradigms. They correspond to the } \\
\text { largest (as an absolute value) difference among the four available (two eyes and two saccade } \\
\text { directions - namely, both directions for the large amplitude paradigms and centripetal directions alone for the } \\
\text { small amplitude paradigms) between short and long ISI for corresponding parameters divided by the average } \\
\text { of the two values. The differences were considered with their signs. }\end{array}$} \\
\hline
\end{tabular}

showed that the MyD mean value was higher than those of the MS group (contrast $1: t 37=3.19$ and $p=0.03$ ) and of the control group (contrast 2 : $t 37=2.88$ and $\mathrm{p}=0.007$ ).

Table 2 Mean (SEM) values for the absolute values of disconjugate duration, peak velocity, and skewness for long ISIs, computed for the myotonic (MyD), multiple sclerosis (MS) and control groups

\begin{tabular}{|c|c|c|c|c|}
\hline $\begin{array}{l}\text { Disconjugate } \\
\text { (right-left eye) } \\
\text { parameter }\end{array}$ & $\begin{array}{l}\text { Target } \\
\text { displacement }\end{array}$ & $\begin{array}{l}\text { Patient } \\
\text { group }\end{array}$ & Mean & SEM \\
\hline \multirow[t]{6}{*}{ Duration } & \multirow[t]{3}{*}{ Small } & MyD & 0.06 & 0.01 \\
\hline & & MS & 0.15 & 0.06 \\
\hline & & Control & 0.09 & 0.02 \\
\hline & \multirow[t]{3}{*}{ Large } & MyD & 0.02 & 0.01 \\
\hline & & MS & 0.08 & 0.05 \\
\hline & & Control & 0.03 & 0.01 \\
\hline \multirow[t]{6}{*}{ Peak velocity } & \multirow[t]{3}{*}{ Small } & MyD & 0.08 & 0.01 \\
\hline & & MS & 0.11 & 0.03 \\
\hline & & Control & 0.06 & 0.02 \\
\hline & \multirow[t]{3}{*}{ Large } & MyD & 0.07 & 0.01 \\
\hline & & MS & 0.09 & 0.02 \\
\hline & & Control & 0.06 & 0.01 \\
\hline \multirow[t]{6}{*}{ Skewness } & \multirow[t]{3}{*}{ Small } & MyD & 0.13 & 0.03 \\
\hline & & $M S$ & 0.15 & 0.04 \\
\hline & & Control & 0.11 & 0.03 \\
\hline & \multirow[t]{3}{*}{ Large } & MyD & 0.41 & 0.08 \\
\hline & & MS & 0.23 & 0.06 \\
\hline & & Control & 0.16 & 0.04 \\
\hline
\end{tabular}

For each subject the disconjugate parameters were computed as the right-left eye values divided by the mean of the two values, for the same saccade direction corresponding to that of the myotonic parameter
The maximal mean values for the short-long ISI difference for saccade peak velocity were invariably positive-that is, peak velocities were usually higher for short than for long ISIs. The mean values for the three groups did not differ significantly either for small $(F 2,37=0.68, \mathrm{p}=0.513)$ or for large $(F 2,37=2.45, \mathrm{p}=0.1)$ amplitudes.

For each subject, we then considered the disconjugate (the difference between the right and the left eye values divided by the mean of these two values) short-long ISI and the disconjugate long ISI values (either with their signs and as absolute values) corresponding to the maximal short-long ISI difference that identified the myotonic parameters. The mean values for all these disconjugate parameters (duration, peak velocity, and skewness) proved to be no larger in the MyD group than in either the MS or the control groups. Table 2 reports the means (SEM) of the disconjugate long ISI values considered with their signs.

We compared the MyD and in the MS groups for abnormal individual maximal short- long ISI differences. Any individual value was considered to be abnormal if it exceeded the mean \pm 2 SD of the control groups, respectively -0.725 and -0.954 for small and large saccade duration; 3.643 and 2.714 for small and large saccade peak velocity; -0.0073 and -0.0062 for small and large saccade skewness.

Patients with MyD showed a greater occurrence of abnormal duration (both for small and large amplitudes) and of abnormal skewness (for small amplitudes only), but not of abnormal peak velocity (figs 1 and 2 ).

Finally, we split the patients with MyD into different subgroups on the basis of MDRS score, and used ANOVA to evaluate whether MDRS score significantly affects any of the myotonic parameters. We found no significant effect. Moreover, we found no correlation between a possible ocular motor myotonia + warm up phenomena and a clinical warm up phenomenon in the skeletal muscles (table 3 ). 


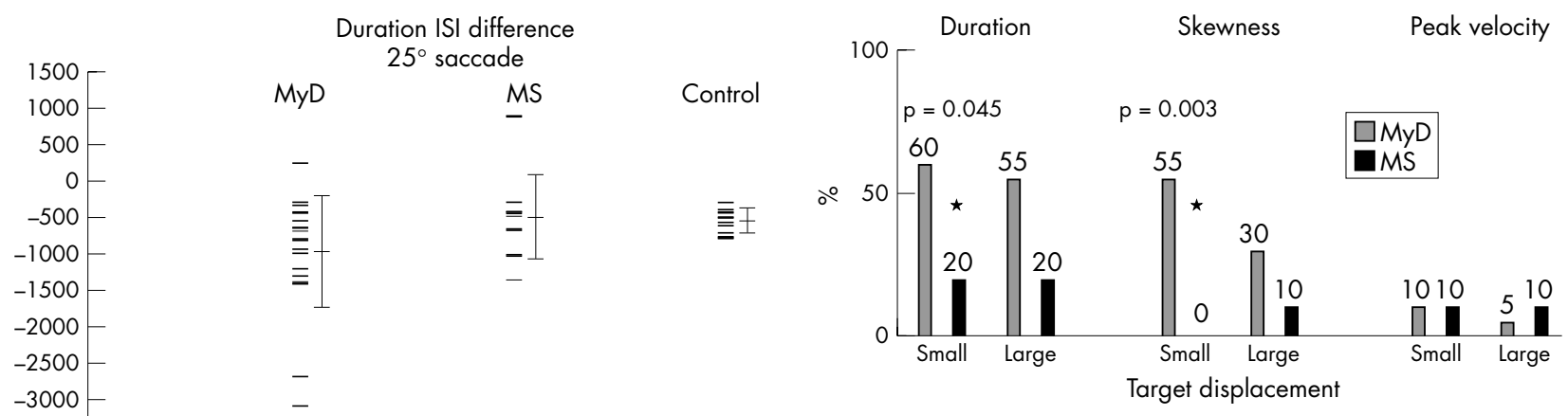

Figure 2 Occurrence of abnormal myotonic parameter values (exceeding the mean +2 SD of the control group) for small and large target displacements in patients with MyD and patients with MD.* Significantly different by Fisher's exact test as shown by the $p$ values above the bars.

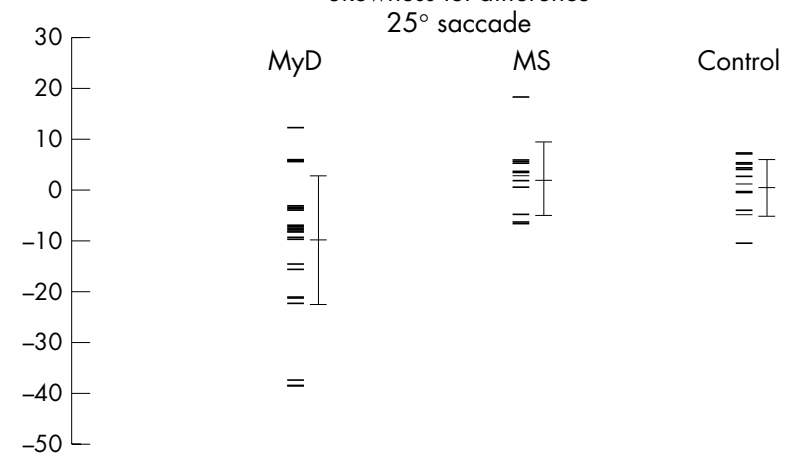

Figure 1 For each subject the figure shows the maximal difference between short and long ISI corresponding (same eye, same direction) duration and skewness values, divided by the mean of these two values (myotonic parameters). The values are $\times 10^{3}$. The subjects are grouped on the basis of diagnosis, and for each group the graph shows a vertical bar corresponding to the mean value (SD).

\section{DISCUSSION}

The abnormalities in ocular movement detectable in our group of patients with MyD are in keeping with those described in previous reports. More specifically, the abnormalities consist of a reduction in saccade peak velocity and an increase in saccade duration.

The main new findings concern the lack of disconjugate (different in the two eyes) abnormalities and the modifications induced on saccade duration and skewness of velocity profile by increasing the interstimulus interval. This finding is explained by the occurrence both of the myotonic and of the warm up phenomenon.

Patients with MyD showed normal eye yoking, as their disconjugate parameters did not significantly differ from those obtained in the control group. None of the patients with MyD presented with clinically evident ocular misalignment or complained of diplopia; nevertheless a subclinical abnormal eye yoking could still be expected as the consequence either of ocular muscle dysfunction (whether myotonic or myopathic) or of CNS impairment.

Within the CNS, cerebellar dysfunction may affect eye yoking. ${ }^{14}$ However, despite the occurrence of CTG expansions in cerebellar tissue,,$^{15}$ patients with MyD do not usually show preeminent clinical and neuroradiological cerebellar involvement, and the saccade abnormalities usually described in patients with MyD are not those attributable to cerebellar dysfunction. Accordingly, our finding that saccades are normally yoked in

\begin{tabular}{|c|c|c|c|c|c|c|c|c|}
\hline Patient No & Age & Sex & MDRS & Warm up & DS & DL & SS & SL \\
\hline 1 & 47 & $\mathrm{~F}$ & 3 & Yes & No & Yes & No & No \\
\hline 2 & 56 & M & 3 & No & No & No & No & No \\
\hline $3^{*}$ & 28 & M & 4 & & No & Yes & No & No \\
\hline $4^{*}$ & 30 & $\mathrm{~F}$ & 4 & & Yes & Yes & No & Yes \\
\hline 5 & 50 & $\mathrm{~F}$ & 4 & No & No & No & Yes & Yes \\
\hline 6 & 44 & M & 4 & Yes & Yes & No & Yes & No \\
\hline 7 & 26 & M & 2 & No & Yes & Yes & No & No \\
\hline 8 & 58 & $\mathrm{~F}$ & 4 & Yes & Yes & Yes & Yes & No \\
\hline 9 & 60 & M & 4 & Yes & Yes & Yes & Yes & No \\
\hline $10 \dagger$ & 27 & M & 3 & Yes & Yes & Yes & Yes & Yes \\
\hline $11 \dagger$ & 21 & $\mathrm{~F}$ & 3 & Yes & Yes & Yes & Yes & Yes \\
\hline 12 & 34 & M & 2 & Yes & Yes & Yes & No & No \\
\hline 13 & 68 & M & 2 & Yes & No & No & No & No \\
\hline 14 & 57 & $\mathrm{~F}$ & 4 & Yes & Yes & No & Yes & No \\
\hline 15 & 15 & $\mathrm{~F}$ & 3 & Yes & No & No & Yes & No \\
\hline 16 & 34 & M & 3 & No & Yes & Yes & Yes & No \\
\hline 17 & 67 & M & 4 & No & No & No & No & No \\
\hline $18 \ddagger$ & 49 & $\mathrm{~F}$ & 2 & Yes & Yes & No & No & Yes \\
\hline $19 \ddagger$ & 21 & $\mathrm{~F}$ & 3 & Yes & Yes & Yes & Yes & Yes \\
\hline 20 & 35 & $\mathrm{~F}$ & 4 & Yes & No & No & Yes & No \\
\hline \multicolumn{9}{|c|}{$\begin{array}{l}\text { * } \dagger \text {, } \neq \text { Patients deriving from the same family. } \\
\text { MDRS, Muscular disability rating scale developed by Mathieu et al. }{ }^{16} \mathrm{MDRS} \text {, ordinal scale of clinical } \\
\text { muscular impairment ranging from } 1 \text { (no impairment) to } 5 \text { (severe proximal weakness, confined to } \\
\text { wheelchair); yes or no, whether the patient's value exceeds the normal limits computed in the control group. } \\
\mathrm{DS} \text { and DL, respectively the duration values for small and large target displacements; SS and SL, respectivel } \\
\text { the skewness values for small and large target displacements. }\end{array}$} \\
\hline
\end{tabular}


A

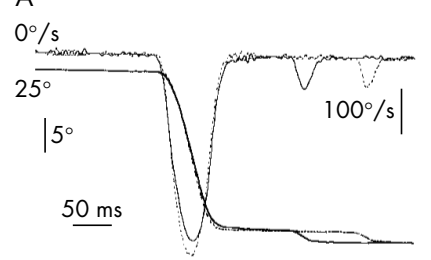

B

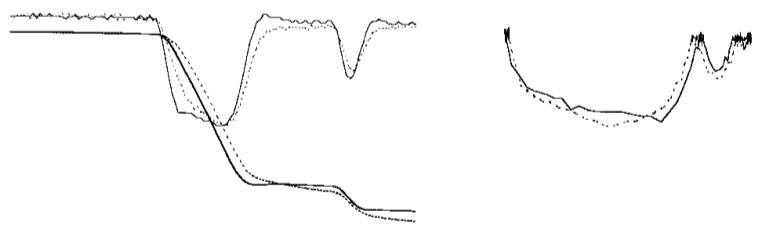

Figure 3 A and $B$ show the velocity (thinner lines) and the position profile (thicker lines) of a leftward centripetal saccade to primary position after the $25^{\circ}$ position has been kept for 1 second (short ISI dotted lines) or for 5 seconds (long ISI continuous lines). A shows a control subject, whereas B shows a patient with myotonic dystrophy. The patient makes a saccade which is slower than the control subject with both ISIs. In the control subject, both the saccade duration and the velocity profile are not significantly modified by the difference in ISI, although the peak velocity is slightly lower for long than for short ISI. By contrast, the patient's saccade reaches the same peak velocity with both ISIs, but with the long ISI the saccade lasts longer; this effect is due to a prolongation of acceleration period, which increases the skewness of the velocity profile. These same points are shown in $C$ and $D$, where saccade velocity is plotted against eye position, starting from $25^{\circ}$ to primary position $\left(0^{\circ}\right)$ during the same respective saccades of $A$ and $B$. The control subject $(C)$ shows the same profile for both ISIs. By contrast, the patient (D) shows a longer acceleration period for long (continuous line) than for short (dotted line) ISI. Note that this does not hold true for corrective saccades.

MyD argues against both cerebellar impairment (as might be expected) and ocular muscle dysfunction.

The last point concerns the detection of an ocular motor myotonic phenomenon. We hypothesised that if it co-occurred with the warm up phenomenon, saccade pacing would presumably modify saccade parameters. Moreover, the two phenomena possibly occurred in a single rather than in all ocular motor muscles, in which case the given saccade modifications could be expected to vary between the different subjects in an eye and direction dependent way. Accordingly, for each subject we considered the largest (as absolute value) ISI difference with its sign between the values available from the two eyes and from the different saccade directions.

Our findings showed that saccade duration and skewness were influenced by the interstimulus interval. In the patients with MyD, prolongation of the stimulus interval led to an increase in saccade duration, without significant modification in saccade peak velocity, and to a reduction in saccade skewness. The reduction derived from an increase in the acceleration duration with respect to the deceleration duration as shown in figure 3. The figure refers to the patient with the largest myotonic skewness, and in this patient the second half of the acceleration period seems to be more prolonged than the first half. However, this asymmetric behaviour was detectable, but less evident, in only three other patients.

These modifications were more significant for small than for large target displacements, and were larger, and occurred more often, in the MyD than in the control or the MS groups.
Moreover, many patients with MyD (15/20 for small target displacements) showed both the maximal increase in duration and the maximal decrease in skewness as occurring in the same eye and in the same saccade direction.

Taken together, these findings may be presumed to derive from a delay in decontracting an extraocular muscle after fixation, in order to make a saccade in the off direction for that muscle (for instance, a delay in decontracting the right lateral rectus to make a leftward saccade starting from a rightward orbital position).

Moreover, the fact that the delay here described occurs more often in the MyD than in the MS group suggests that this phenomenon likely derives from ocular muscle rather than from CNS dysfunction; we hypothesise that the delay may be considered an ocular motor myotonic phenomenon, although we cannot exclude a myopathic origin.

The phenomenon is both slight, as it is not able to affect saccade peak velocity and saccade yoking, and short lasting, as it is less evident for large amplitude and long duration saccades. However, it may provide some insight into the nature of the disorder affecting extraocular and skeletal muscles in myotonic dystrophy.

Authors' affiliations

M Versino, G Sandrini, V Cosi, Fondazione Istituto Neurologico C Mondino, IRCCS, Università di Pavia, Via Palestro 3, 27100 Pavia, Italy M Versino, B Rossi, G Sandrini, V Cosi, Dipartimento di Scienze Neurologiche

G Beltrami, Dipartimento di Informatica e Sistemistica

\section{REFERENCES}

1 Edstrõm L. Dystrophia myotonica. Clinical, pathophysiological and molecular aspects. Scand J Rehabil Med Suppl 1999;39:47-52.

2 Pizzuti A, Friedman DL, Caskey CT. The myotonic dystrophy gene. Arch Neurol 1993;50:1173-9.

3 Morgenlander JC, Massey JM. Myotonic dystrophy. Semin Neurol $1991 ; 11: 236-43$.

4 Thornton C. The myotonic dystrophies. Semin Neurol 1999;19:25-33.

5 Cooper RG, Stokes M, Edwards RH. Physiological characterisation of the "warm up" effect of activity in patients with myotonic dystrophy. J Neurol Neurosurg Psychiatry 1988;51:1134-41.

6 Ter Bruggen JP, Bastiaensen LAK, Tijssen CC, et al. Disorders of eye movement in myotonic dystrophy. Brain 1990;113:463-73.

7 Bollen E, Den Heyer JC, Tolsma MHJ, et al. Eye movements in myotonic dystrophy. Brain 1992;1 15:445-50.

8 Ter Bruggen JP, Tijssen CC, Bastiaensen LAK. Myotonic dystrophy: the natural history of eye movement disorders. Neuroophthalmology 1993;13:85-8.

9 Anastasopoulos D, Kimmig H, Mergner T, et al. Abnormalities of ocular motility in myotonic dystrophy. Brain 1996;1 19:1923-32.

10 Versino M, Romani A, Bergamaschi R, et al. Eye movement abnormalities in myotonic dystrophy. Electroencephalogr Clin Neurophysiol 1998:109:184-90.

11 Shaunak S, Orrell R, Henderson L, et al. Saccades and smooth pursuit in myotonic dystrophy. J Neurol 1999;246:600-6.

12 Davidson SI. The eye in dystrophia myotonica with a report on electromyography of the extra-ocular muscles. $\mathrm{Br} J \mathrm{O}$. phthalmol 1961;45:183-96

13 Hansen HC, Lueck CJ, Crawford TJ, et al. Evidence for the occurrence of myotonia in the extraocular musculature in patients with dystrophia myotonica. Neuroophthalmology 1993;13:17-24.

14 Versino M, Hurko O, Zee DS. Disorders of binocular control of eye movements in patients with cerebellar dysfunction. Brain 1996;1 19:1933-50.

15 Ishii S, Nishio T, Sunohara N, et al. Small increase in triplet repeat length of cerebellum from patients with myotonic dystrophy. Hum Genet 1996;98:138-40.

16 Mathieu J, De Braekeleer M, Prévost C, et al. Myotonic dystrophy: clinical assessment of muscular disability in an isolated population with presumed homogeneous mutation. Neurology 1992;42:203-8. 